\title{
Mutations in 2 distinct genetic pathways result in cerebral cavernous malformations in mice
}

\author{
Aubrey C. Chan,, 1,2 Stavros G. Drakos, ${ }^{1,3}$ Oscar E. Ruiz, ${ }^{4}$ Alexandra C.H. Smith, ${ }^{1,4}$ \\ Christopher C. Gibson, ${ }^{1,5}$ Jing Ling, ${ }^{1}$ Samuel F. Passi, ${ }^{1}$ Amber N. Stratman, ${ }^{6}$ Anastasia Sacharidou, ${ }^{6}$ \\ M. Patricia Revelo, ${ }^{7}$ Allie H. Grossmann, ${ }^{1,7}$ Nikolaos A. Diakos, ${ }^{1}$ George E. Davis, ${ }^{6}$ \\ Mark M. Metzstein, ${ }^{4}$ Kevin J. Whitehead, ${ }^{1,3}$ and Dean Y. Li1,2,3,4

\begin{abstract}
${ }^{1}$ Molecular Medicine Program, ${ }^{2}$ Department of Oncological Sciences, ${ }^{3}$ Division of Cardiology, Department of Medicine, ${ }^{4}$ Department of Human Genetics, and ${ }^{5}$ Department of Bioengineering, University of Utah, Salt Lake City, Utah, USA. ${ }^{6}$ Department of Medical Pharmacology and Physiology, School of Medicine, University of Missouri School of Medicine, Columbia, Missouri, USA.

7Division of Anatomic Pathology, Department of Pathology, University of Utah, Salt Lake City, Utah, USA.
\end{abstract}

\begin{abstract}
Cerebral cavernous malformations (CCMs) are a common type of vascular malformation in the brain that are a major cause of hemorrhagic stroke. This condition has been independently linked to 3 separate genes: Krev1 interaction trapped (KRIT1), Cerebral cavernous malformation 2 (CCM2), and Programmed cell death 10 (PDCD10). Despite the commonality in disease pathology caused by mutations in these 3 genes, we found that the loss of $P d c d 10$ results in significantly different developmental, cell biological, and signaling phenotypes from those seen in the absence of $\mathrm{Ccm} 2$ and Krit1. PDCD10 bound to germinal center kinase III (GCKIII) family members, a subset of serine-threonine kinases, and facilitated lumen formation by endothelial cells both in vivo and in vitro. These findings suggest that CCM may be a common tissue manifestation of distinct mechanistic pathways. Nevertheless, loss of heterozygosity (LOH) for either Pdcd10 or Ccm2 resulted in CCMs in mice. The murine phenotype induced by loss of either protein reproduced all of the key clinical features observed in human patients with CCM, as determined by direct comparison with genotype-specific human surgical specimens. These results suggest that CCM may be more effectively treated by directing therapies based on the underlying genetic mutation rather than treating the condition as a single clinical entity.
\end{abstract}

\section{Introduction}

Cerebral cavernous malformations (CCMs) are common vascular malformations with a prevalence of 1 in 200 to 250 individuals in unselected populations $(1,2)$. CCMs can lead to focal neurological deficits, seizures, and hemorrhagic stroke, but no pharmacologic therapy currently exists (3). CCMs predominantly occur in the central nervous system and are characterized by subclinical bleeding and consequential hemosiderin deposits that are detected by MRI (4). MRI is the primary clinical modality for detection, diagnosis, and management of CCMs. Hemosiderin deposits give CCMs an MRI appearance of a central mass with a dark perilesional halo, whose appearance is nearly diagnostic (pathognomonic) of cavernous malformation (5). Cavernous malformations are characterized by a complex of vascular channels of varying sizes lined by a single layer of endothelial cells without any abnormally large arteries, arterialized veins, or large venous outflow vessels. Although dense fibrillary neuroglial tissue may penetrate the mass, vascular channels are generally arranged in a back-to-back pattern with little or no intervening brain parenchyma. There is often a peripheral margin of gliotic tissue containing hemosiderin-laden macrophages (6).

CCMs can occur sporadically or be inherited in an autosomal dominant pattern. Familial CCM has been linked to heterozygosity for any of 3 genes: Krev1 interaction trapped (KRIT1), Cerebral cavernous malformation 2 (CCM2), and Programmed cell death $10(P D C D 10)(7)$. All 3 proteins bind each other in coimmunoprecipitation experiments on cells overexpressing these proteins,

Conflict of interest: The University of Utah seeks to commercialize this technology and has filed patent applications related to this manuscript.

Citation for this article: J Clin Invest. 2011;121(5):1871-1881. doi:10.1172/JCI44393. leading to the hypothesis that they function as a complex to affect a common signaling mechanism (8). Both Krit 1 and $\mathrm{Ccm} 2$ are required for proper connection of the developing heart with the aorta to establish circulation in the mouse embryo $(9,10)$. Mice lacking either Krit1 or Ccm2 fail to form a lumenized first branchial arch artery to link the heart and aorta; as a result, mice lacking either gene die at the same age. The requirement for Ccm2 is endothelial autonomous $(9,11)$. In the endothelium, both KRIT1 and CCM2 suppress the activity of the small GTPase RhoA (9, 12). Loss of either gene leads to RhoA activation and signaling through Rho kinase (ROCK) resulting in increased actin stress fibers, impaired cell-cell interactions, and increased vascular permeability $(9,12,13)$. These defects can be reversed in cell culture and in mice with inhibitors of RhoA including HMG-CoA reductase inhibitors (statins) $(9,12)$.

A recent report suggested a similar mechanism of Rho activation for PDCD10 (14). However, other studies have suggested a different cell-signaling role for PDCD10. While KRIT1, CCM2, and PDCD10 all occupy the cytoplasmic compartment of the cell, unique subcellular localization for each has been described, including nuclear localization of KRIT1 (15) and Golgi localization of PDCD10 (16). Each protein has also been found to have unique binding partners (17-19). PDCD10's binding partners include members of the germinal center kinase III (GCKIII) subfamily of serine-threonine kinases with homology to yeast sterile-20 (STE20) kinase (16, 20-23). Furthermore, the clinical features of CCM in KRIT1, CCM2, and $P D C D 10$ families have important differences $(24,25)$, with PDCD10 resulting in the most severe disease $(24,26)$. It is possible that CCM disease is the common result of multiple unique mechanisms and may require unique therapeutic strategies to target the underlying disturbed cellular and signaling pathways. 


\begin{tabular}{|c|c|c|c|c|}
\hline Cross & & Pdcd10+/- & Pdcd10+- & \\
\hline Genotype & Pdcd10 $+/+$ & Pdcd10+/- & Pdcd10-/- & Total litters \\
\hline No. of progeny & & & & \\
\hline E7.5 & 75 & 114 & 52 & 27 \\
\hline E8.0 & 9 & 13 & $\mathrm{OA}^{\mathrm{A}}$ & 4 \\
\hline E8.5 & 27 & 45 & $\mathrm{OA}^{\mathrm{A}}$ & 13 \\
\hline E9.0 & 1 & 5 & 0 & 1 \\
\hline$>\mathrm{E} 9.0$ & 7 & 8 & 0 & 3 \\
\hline
\end{tabular}

The numbers of normal-appearing and viable offspring by genotype in matings between $\mathrm{Pdcd} 10^{+/-}$parents are shown. ${ }^{\mathrm{A}}$ Ages at which growtharrested embryos could be obtained.

Though the signaling and cellular mechanisms associated with the 3 known CCM proteins may be different, a common genetic mechanism of loss of heterozygosity (LOH) has been suggested for familial CCM disease (27-29). Familial CCM is more aggressive than sporadic disease, with an earlier age of onset, increased risk of hemorrhage and seizure, and an increased number of lesions (3, 30-32). These observations have led to the hypothesis that CCM disease occurs by the Knudson 2-hit mechanism, similar to retinoblastoma (33). There is limited evidence from human pathologic specimens to support an association of $\mathrm{LOH}$ with CCM lesions. In a series of challenging experiments, a number of investigators have identified biallelic mutations, 1 somatic and 1 germline, in the endothelium of a subset of patient samples $(27,28,34)$. While $\mathrm{LOH}$ could not be confirmed for a number of samples, a total of 4 cases have been described for KRIT1 and 1 case each for CCM2 and PDCD10. Mice heterozygous for Krit 1 or $\mathrm{Ccm} 2$ do not develop cavernous malformations, and no examples of secondary somatic mutations have been reported $(35,36)$.

To determine whether endothelial LOH is not simply associated with, but is causative for, CCM pathology, requires an animal model with a controlled genetic mutation that can be directed and detected in a tissue-specific manner. In this work, we use druginducible, tissue-specific strains of Cre recombinase to target conditional null alleles of the CCM genes to test directly the 2-hit hypothesis for cavernous malformations. We demonstrate that Pdcd10 differs substantially from Ccm2 in development, cell biology, and signaling, yet $\mathrm{LOH}$ is the common genetic mechanism to cause CCMs in both genotypes. These findings suggest that PDCD10 influences different endothelial signaling pathways from KRIT1/CCM2 to lead to a common histopathology and imply that medical treatment to stabilize familial CCM may need to be developed and evaluated in a genotype-specific manner.

\section{Results}

Loss of Pdcd10 results in embryonic lethal phenotypes distinct from loss of Krit 1 or Ccm2. To determine the role of $P d c d 10$ in development and disease, we developed a conditional null allele, $P d c d 10^{f l o x}$, in which exons 4-8 are flanked with LoxP sites (Supplemental Figure 1; supplemental material available online with this article; doi:10.1172/ JCI44393DS1). This strategy also allowed us to generate a constitutive null allele, $P d c d 10^{-}$, by crossing mice carrying $P d c d 10^{f l o x}$ with a mouse strain expressing Cre recombinase in the germline (37). To characterize the role of $P d c d 10$ in development, we intercrossed $P d c d 10^{+/-}$mice and harvested embryos at varying stages of devel- opment. Surprisingly, we found that mice lacking Pdcd10 died at a much earlier age than those deficient for either Krit1 or Ccm2. Whereas Krit1 and Ccm 2 mice show growth arrest at E9.0 and die at E11 (9-11), the loss of Pdcd10 leads to growth arrest at E8.0, after gastrulation, but prior to the onset of circulation or the requirement for cardiovascular function (Table 1 and Supplemental Figure 1).

An earlier requirement for $P d c d 10$ than Krit 1 or $C c m 2$ in development does not preclude a shared role in the vascular system. Mice with endothelial loss of $\mathrm{Ccm} 2$ phenocopy the constitutive null mutant $(9,11)$, so we sought to determine whether mice with endothelial loss of $P d c d 10$ would also recapitulate the $C \mathrm{~cm} 2$ phenotype. Using the endothelial Tie2-Cre driver, the same Cre driver that we used to ablate $C \mathrm{~cm} 2$ (9), we found that although mice with endothelial loss of $P d c d 10$ ( $P d c d 10^{\text {flox } /-}$;Tie2-Cre) did not survive to birth, they had patent branchial arch arteries, a developed circulatory system, and were indistinguishable from control littermates at E9.5 (Supplemental Figure 2). Instead, we found that loss of Pdcd10 in the endothelium leads to progressive enlargement of the cardinal vein and other veins of the rostral embryo at E11.5 (Figure 1). Venous enlargement was not due to abnormal cardiac structure or function (Figure 1 and Supplemental Figure 2), nor were defects observed in arteries of the embryo (Figure 1 and Supplemental Figure 2). Death occurred due to hemorrhage from venous rupture by E13.5 (Table 2). Additionally, mice with loss of Pdcd10 in neural and glial tissues induced by Nestin-Cre showed no vascular or any other obvious defects and were born alive (Supplemental Figure 2 and Supplemental Table 1). These observations suggest that $P d c d 10$ is required in the endothelium for control of venous size and integrity, yet $P d c d 10$ differs from $C c m 2$ in that it is not required for the establishment of circulation.

Loss of PDCD10 does not affect RhoA signaling but results in lumen formation defects. To explore the role of PDCD10 in endothelial cells, we depleted PDCD10 in human dermal microvascular endothelial cells (HMVEC) with siRNA (Supplemental Figure 3). Whereas the loss of CCM2 leads to an increase in actin stress fibers as a result of RhoA activation $(9,12,13)$ and phosphorylation of myosin light chain- 2 by Rho kinase (Figure 2), we observed none of these indicators of RhoA activation with the loss of PDCD10 (Figure 2). Thus, the role of PDCD10 in endothelial cell biology and signaling differs from that of CCM2.

Having found that PDCD10's function in development differs from that of CCM2, we sought to identify defects associated with PDCD10 depletion in assays relevant to vascular development. PDCD10-depleted HUVECs showed defective function in a 3D angiogenesis assay. Endothelial cells plated in a collagen matrix spontaneously organize into complex multicellular capillary-like networks with lumens (38), but cells depleted of PDCD10 failed to organize themselves into a lumenized network (Supplemental Figure 4, A and B). We explored potential downstream signaling pathways using this assay. A growing body of evidence suggests that PDCD10 interacts with the GCKIII subfamily of serine-threonine kinases $(16,21-23)$; we used siRNA to deplete cells of each of the subfamily members (Supplemental Figure 3). We observed no effect on lumen formation with the knockdown of STK25, STK24, or MST4 in HUVECs (Supplemental Figure 4C).

Pdcd10 functionally associates with GCKIII in lumen formation. Because the GCKIII family members may be functionally redundant, we sought to validate the importance of the PDCD10-GCKIII interaction in vivo and in a simpler genetic model. We chose the fruit fly, Drosophila melanogaster, in which the GCKIII family is represented by 

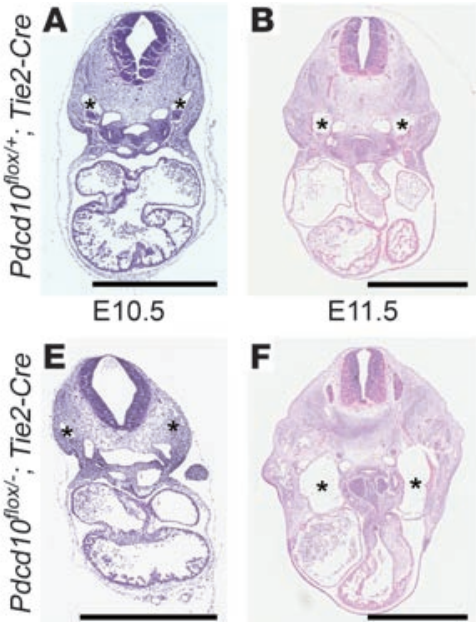

E10.5

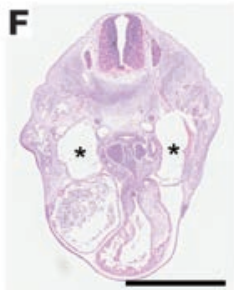

E11.5

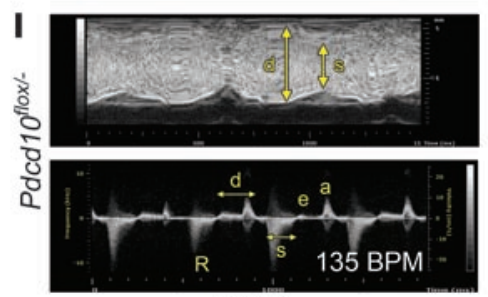

E11.5
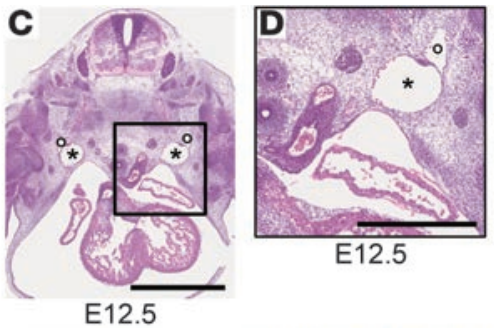

$\mathrm{E} 12.5$

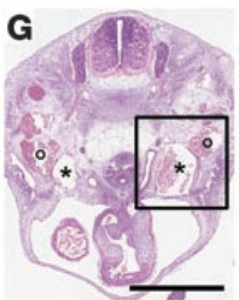

$\mathrm{E} 12.5$

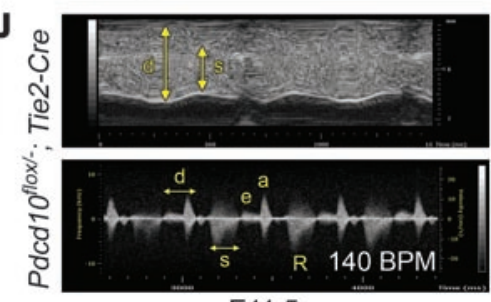

E11.5

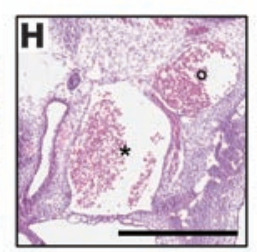

$\mathrm{E} 12.5$

show lack of lumenization in multiple tracheal tubes (Supplemental Figure 4E). The specificity of this effect is confirmed, as it could be rescued by coexpression of Drosophila Pdcd10 in RNAi-expressing cells (Supplemental Figure 4E). Notably, coexpression of the 18-amino acid deletion form of Drosophila Pdcd10, which does not bind to GCKIII, fails to rescue this phenotype (Supplemental Figure 4E). We used the same RNAi strategy to inactivate GCKIII (44) in the developing tracheal system. Tracheal tubes lacking GCKIII exhibit failure of lumenization (Supplemental Figure 5), and the phenotype appears very similar to that of

a single protein, GCKIII. This model avoids the complexity of human or mouse, which has 3 GCKIII kinases, or zebrafish, which has 2 orthologs for $P d c d 10,2$ orthologs for STK25, and single orthologs for STK24 and MST4 (23). Furthermore, the Drosophila genome contains a single ortholog of PDCD10, but no orthologs for KRIT1 or CCM2. Thus, Drosophila represents a simple model organism for studying Pdcd10-specific biology. To ensure that the biochemical interaction between PDCD10 and GCKIII was conserved in Drosophila, we performed immunoprecipitation assays with the Drosophila proteins. Drosophila Pdcd10 binds to GCKIII (Supplemental Figure 4D) in a manner analogous to that of human, mouse, or zebrafish protein $(21,23)$. A human disease PDCD10 mutation exists, which deletes 18 amino acids crucial for PDCD10-GCKIII binding (21). Removal of the analogous 18 amino acids from the Drosophila Pdcd10 protein abrogated binding to GCKIII (Supplemental Figure 4D).

Whereas Drosophila do not develop a vascular system, they do form a branched, lumenized network of tubes in the tracheal (respiratory) system. This epithelial network requires coordinated cell-cell interactions and specialized cell-cell junctions, analogous to the mammalian vascular system $(39,40)$. To determine the necessity of Pdcd10 in developing fly tracheal tubes, we expressed a dsRNA directed against $P d c d 10$ (41) under the control of the tracheal-specific Breatbless promoter (42) using the GAL4-UAS system (43). We found that tracheal tubes lacking Pdcd10 grow and branch normally, but fail to lumenize and fill with air (Supplemental Figure 5), indicating that Pdcd10 is required for normal lumen formation in fly tracheal tubes as it is in human endothelial tubes. This effect is highly penetrant; nearly all dsRNA-expressing flies

\section{Table 2}

Loss of $P d c d 10$ in the endothelium leads to embryonic death after E12.5

\begin{tabular}{|c|c|c|c|c|}
\hline \multirow{2}{*}{$\begin{array}{l}\text { Cross } \\
\text { Genotype }\end{array}$} & \multicolumn{4}{|c|}{ Pdcd10 $0^{f l o x / f l o x} \times$ Pdcd10+-;Tie2-Cre } \\
\hline & Pdcd10flox/+ & Pdcd10tlox/- & Pdcd10 flox/+;Tie2-Cre & Pdcd10flox/-;Tie2-Cre \\
\hline No. of progeny & & & & \\
\hline E9.5 & 14 & 14 & 7 & 11 \\
\hline E12.5 & 13 & 28 & 16 & 16 \\
\hline E15.5 & 8 & 8 & 20 & 0 \\
\hline P21 & 26 & 20 & 20 & 0 \\
\hline
\end{tabular}

Live offspring by genotype at embryonic and postnatal time points resulting from matings between Pdcd10 flox/flox and Pdcd10+/-;Tie2-Cre parents. 
A Control siRNA

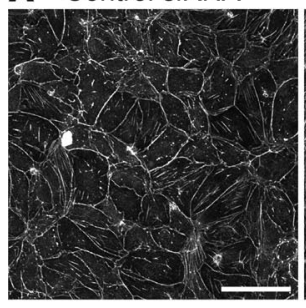

B

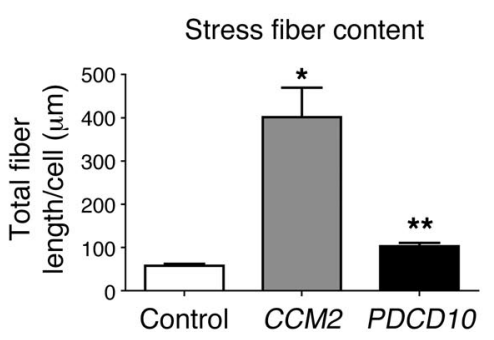

CCM2 SiRNA

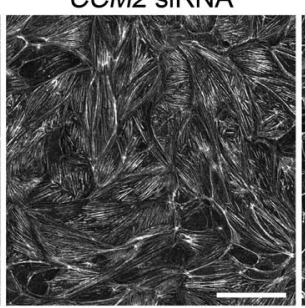

c

\section{PDCD10 siRNA}

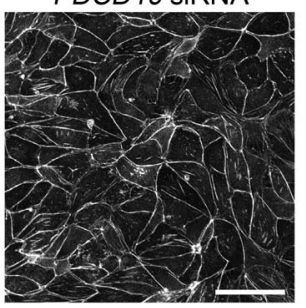

C

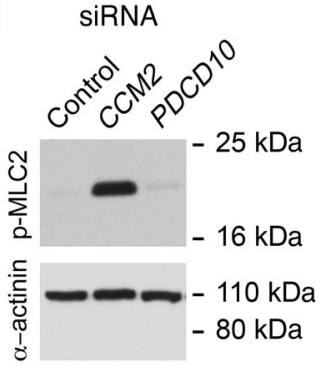

Figure 2

PDCD10 differs from CCM2 in downstream signaling. (A) Phalloidin staining of human microvascular endothelial cells treated with siRNA directed against CCM2, PDCD10, or nonsense control. (B) Quantification of stress fiber response of HMVEC cells. Stress fiber content is determined by adding the total length of stress fibers divided by the total number of cells. Results indicate mean \pm SEM and are representative of at least 3 independent experiments. ${ }^{*} P<0.001$ versus control or $P D C D 10 ;{ }^{*} P=$ NS versus control. (C) Immunoblot for phospho-myosin light chain-2 (with $\alpha$-actinin immunoblot as a loading control). Results are representative of 3 independent experiments. Scale bars: $100 \mu \mathrm{m}$. to the same human disease. Limited human genetics suggested an association between $\mathrm{LOH}$ and CCM disease, so we investigated whether $P d c d 10$ and $C c m 2$ share this genetic mechanism for causing disease. To control the timing of the endothelial "second-hit," we used a drug-inducible Cre strain under the control of the PDGF-B promoter. This $P D G F b-i C r e E R^{T 2}$ strain expresses a Cre recombinase activated only after administration of the drug tamoxifen. Consistent with the original report describing this recombinase (37), we found that the administration of tamoxifen on the first postnatal day (P1) led to efficient, endothelial-specific recombination throughout the entire brain vasculature (Supplemental Figure 6). The induction of endothelial LOH of either $P d c d 10$ or $C \mathrm{~cm} 2$ by this Cre at birth resulted in CCMs in mice as early as 1 month of age (Figure 3). Induction of endothelial LOH of either $P d c d 10$ or $\mathrm{Ccm} 2$ resulted in a spectrum of vascular malformations, from capillary telangiectasias, to isolated caverns, to multiple back-to-back caverns with thrombosis, hemorrhage, and formation of secondary channels (Supplemental Figure 7). Loss of protein product via LOH was confirmed by antibody staining (Supplemental Figure 8). The retinal vasculature is another location for human CCMs, and murine cavernous malformations were also observed in mouse retinal vasculature (Supplemental Figure 9). To formally prove LOH in the endothelium of these CCMs, we performed laser capture microdissection to obtain tissue-specific DNA as previously done in human studies of CCM (28). We found that DNA from lesion endothelium had lost the conditional allele for either $P d c d 10$ or $\mathrm{Ccm} 2$, confirming $\mathrm{LOH}$, whereas this allele could still be detected in adjacent neuronal tissue (Figure 3). In contrast, loss of Ccm 2 in neural tissues using Nestin-Cre did not result in the development of vascular malformations (Supplemental Figure 10).

We characterized the histopathologic features of cavernous malformations in mice with induced endothelial loss of either Pdcd10 (Figure 4) or Ccm2 (Figure 5). We compared mouse CCMs with surgical specimens from CCM patients with germline mutations of either PDCD10 (Figure 4) or CCM2 (Figure 5). Cavernous malformations in these induced mouse models share all of the key histologic features of CCM lesions with human specimens. Furthermore, both $P d c d 10$ and $C \mathrm{~cm} 2$ resulted in identical pathologic findings for all of these defining characteristics in both human and mouse (Table 3). Mouse specimens showed numerous

\section{Figure 3}

Cavernous malformations result from $\mathrm{LOH}$ of either Ccm2 or Pdcd10. (A) Cavernous malformation (arrow) shown in an H\&E-stained section of brain cerebrum from a mouse with induced endothelial knockout of $\mathrm{Ccm} 2$. (B) Confirmation of $\mathrm{LOH}$ of $\mathrm{Ccm} 2$ in 2 mice with loss of $\mathrm{Ccm} 2^{\text {flox }}$ allele by PCR, compared with Pdcd10 wild-type allele as a control. (C) Cavernous malformations (arrows) and a less complex telangiectasia (arrowhead) shown in an H\&E-stained section of brain cerebrum from a mouse with induced endothelial knockout of Pdcd10. (D) Confirmation of $\mathrm{LOH}$ of Pdcd10 in 2 mice by PCR with loss of Pdcd10 flox allele compared with $\mathrm{Ccm} 2$ wild-type allele as a control. Samples in $\mathbf{B}$ and $\mathbf{D}$ were obtained via laser capture microdissection of sectioned mouse brains. Scale bars: $1 \mathrm{~mm}$.
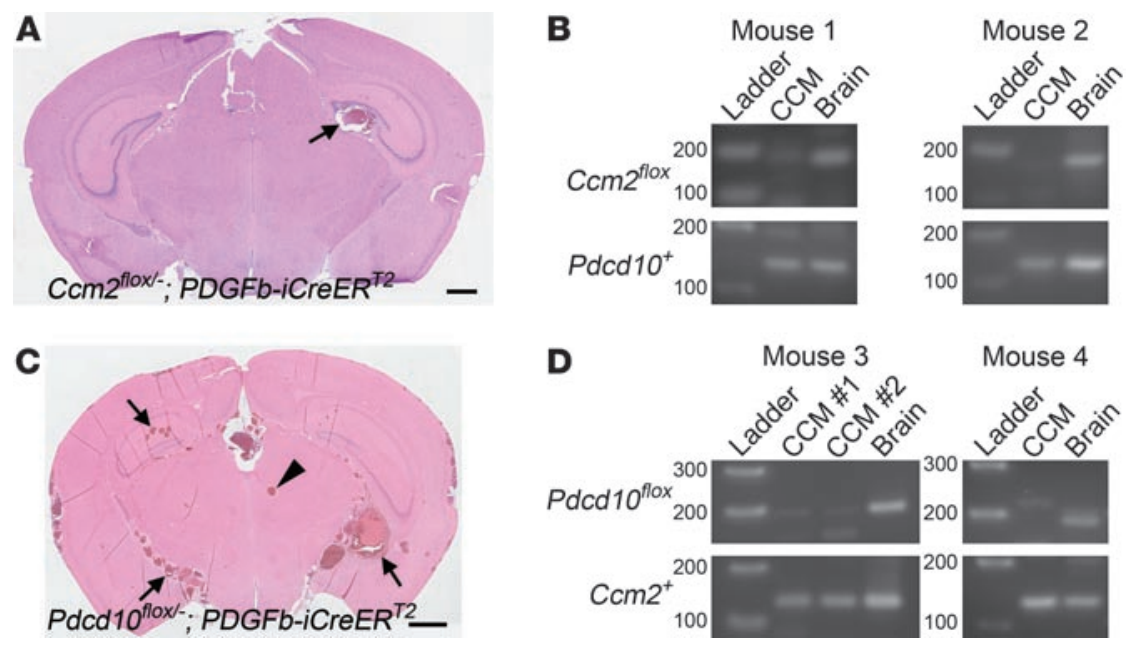

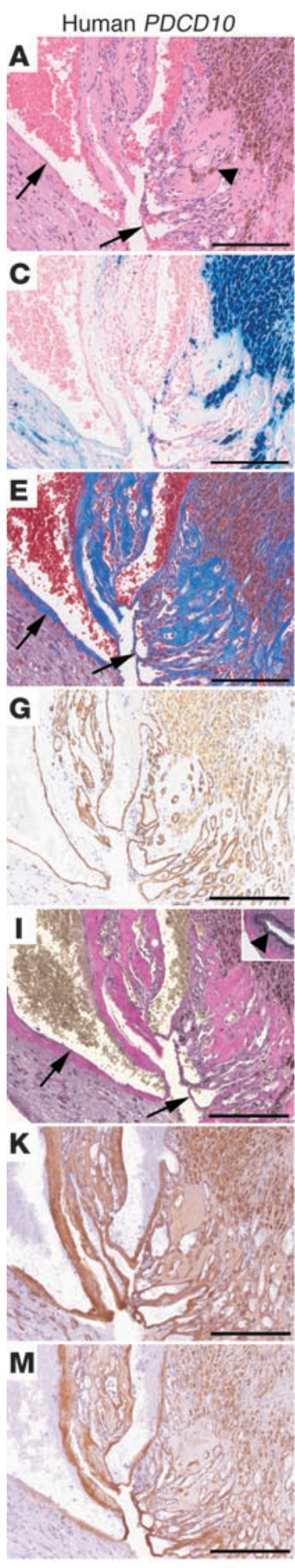

\section{Figure 4}

Pathologic analysis of mouse and human PDCD10-associated CCM. Paired analysis of histologic sections with human tissue on the left and mouse on the right. (A and B) H\&E staining revealing back-to-back vascular channels (arrows) and hemosiderin pigment (arrowheads) in surrounding tissues. (C and $\mathbf{D}$ ) Iron (blue) detected by Prussian blue stain highlights hemosiderin deposits in macrophages and surrounding brain tissue. (E and F) Fibrous matrix deposits (blue) identified by Masson's trichrome staining with fibrous tissue surrounding vascular channels (arrows) and in surrounding gliotic brain. ( $\mathbf{G}$ and $\mathbf{H})$ Endothelial staining for CD34 (G) or CD31 $(\mathbf{H})$ is positive in the cells lining the channels. (I and J) Elastin staining shows that vascular channels lack elastic laminae (arrows) unlike normal vessels of similar caliber (arrowhead, inset in I). The fibrous matrix surrounding channels includes laminin ( $\mathbf{K}$ and $\mathbf{L}$ ) and collagen IV (M and $\mathbf{N}$ ). Scale bars: $200 \mu \mathrm{m}$.

gliosis, trichrome staining, elastin, laminin, and collagen type IV, and these features were all identical between mouse and human lesions (Figure 4, E and N, Figure 5, E and N, and Table 3).

We also examined mouse lesions at the ultrastructural level using transmission electron microscopy. Murine cavernous malformations showed endothelial cells lining vascular channels with associated basal lamina (Figure 6A). Some of the larger channels showed segmental multilayering (lamellated appearance) of the basal lamina (Figure 6B). The most dilated channels showed focally marked attenuation of the endothelial cells (Figure 6C); however, tight junctions were identified between cells, and definitive gaps were not seen. Connective tissue composed mainly of collagen fibers separated the vascular channels (Figure 6D). Pericytes or astrocytic foot processes were missing, as seen in human lesions. Foci of mononuclear inflammatory cells were also seen (Figure 6E), including hemosiderin-laden macrophages (Figure 6F). Ultrastructural analysis of murine CCM lesions was similar to that described in human CCMs (45), further solidifying the fidelity of the mouse model to the human disease.

Murine cavernous malformations can be detected and followed noninvasively by MRI. MRI is used to document the natural history of human cavernous malformations and will be necessary for prospective therapeutic trials in both mice and humans. We thus employed monthly live MRI studies to follow the onset and progression of disease in mice until at least 6 months of age (Figure 7). As in humans (24), mice lacking $P d c d 10$ had an earlier onset of disease, with a more severe phenotype than Ccm2. All Pdcd10 mice studied at 1 month of age had lesions, whereas no $\mathrm{Ccm} 2$ mice had yet developed lesions by 2 months of age (Figure $7 \mathrm{~K}$ ). Mice with $P d c d 10$ mutations had a greater disease burden when assessed by total lesion burden (Figure 7L) or by number of complex lesions (increased signal intensity within the lesion; Figure 7M). Cavernous malformations were fully penetrant in mice with $\mathrm{Ccm} 2 \mathrm{LOH}$ at 6 months of age and most had lesions at 4 months, at which time the disparity between the genotypes began to narrow. This parity reflected not only the increased burden of disease in $\mathrm{Ccm} 2$, but also the onset of death in the most severely affected Pdcd10 mice. Mice of both genotypes began to die of hemorrhage, but mortality was greater in mice with LOH for Pdcd10 (Figure 7N), a finding that reflects the reported experience in humans (24).

Figure 5, A and B), some of them with organized thro Immunohistochemical stains for endothelium highlighted endothelial cells in the channels, with focal attenuation or loss of endothelial cells in larger channels (Figure 4, G and H, and Figure 5, G and H). Iron stain highlighted the presence of hemosiderin-laden macrophages and hemosiderin in the wall of the channels as well as in the periphery and brain tissue (Figure 4, C and D, and Figure 5, C and D). Additionally, we also examined

\section{Discussion}

Although CCM has been associated with mutations in 3 distinct loci, it has been clinically treated as a single pathophysiologic entity. In this manuscript, we describe for what we believe is the first time the pathologic features of CCM in a genotype-specific manner. We 

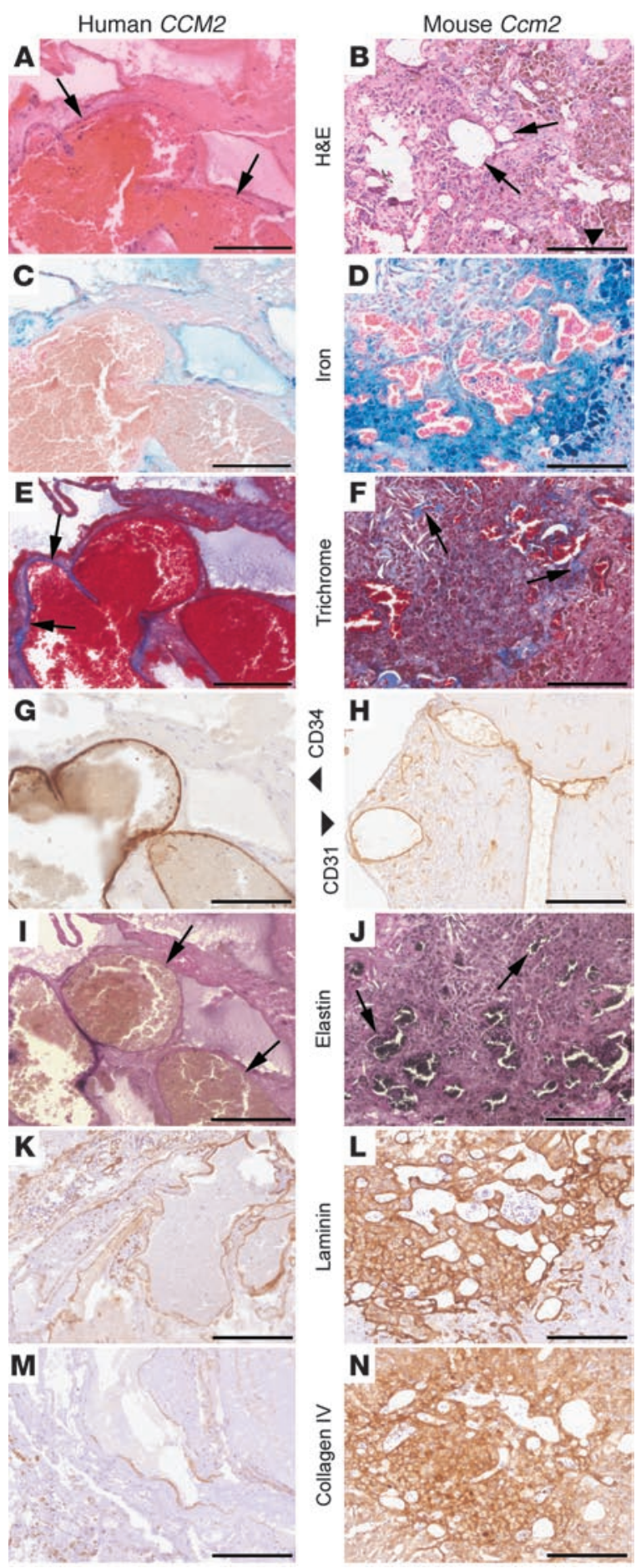

find that mutations of both PDCD10 and CCM2 result in a common pathologic expression of disease in both humans and mice. Surprisingly, this common disease endpoint does not constitute proof of a common disease mechanism. Whereas our previous work and the work of others found that $\mathrm{Ccm} 2$ and Krit 1 play similar roles in embryonic development, in vitro cell biology, and cell signaling $(9,10,12,13)$, these roles differ from those we observe with Pdcd10. Unlike Krit1 and Ccm2, Pdcd10 is not required for development of the branchial arch arteries that connect the heart to the aorta.

\section{Figure 5}

Pathologic analysis of mouse and human CCM2-associated CCM. Paired analysis of histologic sections with human tissue on the left and mouse on the right. (A and $\mathbf{B}$ ) H\&E staining revealing back-to-back vascular channels (arrows) and hemosiderin pigment (arrowhead) in surrounding tissues. (C and $\mathbf{D}$ ) Iron (blue) detected by Prussian blue stain highlights hemosiderin deposits in macrophages and surrounding brain tissue. (E and F) Fibrous matrix deposits (blue) identified by Masson's trichrome staining with fibrous tissue surrounding vascular channels (arrows) and in surrounding gliotic brain. ( $\mathbf{G}$ and $\mathbf{H}$ ) Endothelial staining for CD34 (G) or CD31 $(\mathbf{H})$ is positive in the cells lining the channels. (I and $\mathbf{J}$ ) Elastin staining shows that vascular channels lack elastic laminae (arrows). The fibrous matrix surrounding channels includes laminin ( $\mathbf{K}$ and $\mathbf{L}$ ) and collagen IV ( $\mathbf{M}$ and $\mathbf{N}$ ). Scale bars: $200 \mu \mathrm{m}$.

Rather, Pdcd10 has an essential, nonendothelial role in development not shared with Krit 1 or $\mathrm{Ccm} 2$ as well as an essential function in venous maturation. We further observe differences in cell biology and signaling between PDCD10 and KRIT1 or CCM2; whereas the loss of KRIT1 or CCM2 leads to RhoA activation, increased Rho kinase activity, myosin light chain phosphorylation, and actin stress fiber formation $(9,12,13)$, we do not observe activation of this signaling pathway in cells depleted of PDCD10. Instead, we found that PDCD10 signals primarily through the GCKIII family of kinases.

The similarity in human CCM disease caused by mutations in KRIT1, CCM2, and PDCD10 have led to an assumption that the proteins encoded by these genes function in a common signaling pathway. This assumption has been supported by experimental evidence showing binding between ectopically expressed, epitope-tagged proteins $(7,8)$. However, the complexities of signaling pathways and pathophysiology allow for multiple mechanisms to converge on a common disease phenotype (Figure 8A). An example is hypertrophic cardiomyopathy, which was considered 1 disease until molecular genetics revealed that 2 different mechanisms (sarcomere function or metabolism), each affected by distinct genes, both result in pathologic hypertrophy (Figure $8 \mathrm{~B}$ and ref. 46). We propose that a similar scenario is involved in the pathogenesis of CCM (Figure 8C).

There is controversy concerning the signaling pathways affected by PDCD10. Several reports suggest an essential role for binding GCKIII family serine-threonine kinases (16, 21-23). However, a recent characterization of mice carrying a different conditional allele of Pdcd10 showed that the loss of Pdcd10 in endothelial cells substantially blocks VEGFR2 signaling and inhibits the earliest stages of developmental angiogenesis (47). The implication of VEGFR2 signaling through MAP kinases agrees with a previous report linking GCKIII kinases and ERK signaling (20). Our data contrast with these reports; we observe that the absence of Pdcd10 in vivo leads to a localized vascular defect at a much later developmental stage, inconsistent with a panendothelial block of VEGF signaling. In cell culture, we have not found any interaction between PDCD10 and VEGF or ERK signaling (Supplemental Figure 11). We suspect that this disparity may be due to a difference in knockout strategy; however, our confidence in our allele is bolstered by our ability to induce CCM disease. Our data support the model that PDCD10 signals through the GCKIII family kinases, as we have observed in human endothelial cells and in Drosophila that PDCD10 binds to GCKIII family kinases and both are required for lumen formation. Previous reports have further suggested that GCKIII signals through RhoA to converge 
Table 3

Pathologic findings in human and murine CCMs

\begin{tabular}{lcccc} 
Genotype & Human CCM2 & Murine Ccm2 & Human PDCD10 & Murine Pdcd10 \\
Trichrome & + & + & ++ & ++ \\
Iron & + & + & ++ & ++ \\
Elastin & - & - & - & - \\
CD34 & + & NA & + & NA \\
CD31 & NA & + & NA & + \\
Laminin & + & + & + & + \\
Collagen IV & + & + & + & + \\
\hline
\end{tabular}

The pathologic findings compared for human and murine CCMs associated with mutations of either CCM2 or PDCD10.

with KRIT1/CCM2 signaling (23). By direct comparison of Ccm2 and $\mathrm{Pdcd} 10$ in mouse and cell biology, however, our data suggest that PDCD10 signaling is distinct from the CCM2-RhoA axis.

The nature and severity of disease in familial forms of CCM in comparison with sporadic CCM suggested a genetic mechanism consistent with Knudson's 2-hit hypothesis (33): LOH for a CCM gene induces lesions. Limited evidence to support this theory has come from a few human surgical samples amid multiple cases in which the second genetic hit could not be found $(27,28)$. Further supportive evidence comes from mice with heterozygous mutations for Krit1 or C cm2 that have been mated into strains with high rates of spontaneous mutations. Mice heterozygous for Krit1 develop CCM lesions on either a p53- or Msh2-null background, whereas mice heterozygous for $\mathrm{Ccm} 2$ develop lesions only on the $\mathrm{p} 53$-, but not the Msh2-null background $(35,36)$. Whereas these models provide suggestive evidence of $\mathrm{LOH}$ and employ stochastic events to induce CCM formation, $\mathrm{LOH}$ was not demonstrated at either locus, nor can the $\mathrm{LOH}$ hypothesis be supported for all CCM genes, as $\mathrm{Ccm} 2^{+/-} ; \mathrm{Msh}^{-1-}$ mice do not develop CCMs. These models also do not rule out a role for mutations in other, non-CCM genes and do not control tissue specificity of mutation. The penetrance of CCMs in these models is incomplete, complicating the use of these models in prospective trials to study therapeutics or natural history of CCM disease. Concurrent development of neoplasms in both the p53- and the Msh2-null backgrounds also adds confounding physiological stressors and increases the mortality of the animals. In contrast, we employ a strategy that allows direct testing of the
LOH mechanism in CCM disease. Using an inducible Cre-recombinase, we have targeted gene-specific LOH for both $P d c d 10$ and $C c m 2$ to the endothelium of mice. In the case of both genes, we have found that $\mathrm{LOH}$ is sufficient to cause a fully penetrant CCM phenotype that recapitulates every key pathologic and radiologic hallmark of human disease.

Much work remains to translate the observations and insights regarding disease signaling mechanisms into viable therapeutic strategies in patients. This work underscores the importance of carefully considering disease mechanisms in a genotype-specific manner. The availability of faithful, genotype-specific, and highly penetrant mouse models of CCM disease unlocks the tremendous opportunities to study the natural history of lesion genesis and progression as well as opportunities for preclinical testing of therapeutic interventions. In order for mouse models of CCM disease to be useful in informing human studies, the same tools used to follow patients with CCM need to be developed for serial observation of affected mice. We have demonstrated that noninvasive MRI of live mice, the same modality used to follow CCM in humans, can detect and follow murine CCMs. Although our mouse models share a measurable mortality, as with humans, they are compatible with prolonged survival and serial, noninvasive observation. The ability to follow these mice noninvasively over time is a crucial prerequisite for judging the effectiveness of any preclinical therapeutic strategy in the future and for testing the timing and intensity of $\mathrm{LOH}$ required for lesion formation. Our mouse models of CCM phenocopy human disease closely, supplanting previously available surrogate phenotypes, and are a powerful new tool in the armamentarium to decipher and combat CCM disease.

\section{Methods}

Mouse strains. The $\mathrm{Ccm} 2$ conditional knockout mice have been previously described (9). A construct for the conditional allele of Pdcd10 was derived from genomic sequence obtained from a BAC clone (RP22 library; Invitrogen). The construct extended from a SacII site $5^{\prime}$ of exon 4 through an ApaI site $3^{\prime}$ of exon 8. The construct contained inserts as outlined in Supplemental Figure 1, including LoxP sequences at a KasI site just $5^{\prime}$ of exon 4 and at a EcoRI site just $3^{\prime}$ of exon 8 . All mice were backcrossed into the C57BL/6J strain. Experiments performed prior to the fifth cross were performed with littermate controls. LacZ reporter mice (R26R1), Tie2-Cre

\section{Figure 6}

Ultrastructural findings in murine cavernous malformations. (A) Dilated vascular channels are lined by endothelial cells (arrowheads) with associated basal lamina (arrows) (B) Occasional channels have segments with a multilayered appearance (arrows indicate lamellae of endothelium with basal laminae). Tight junctions appear intact (arrowhead). (C) Focal areas of endothelial attenuation are observed (arrow) without apparent gaps or disruption of tight junctions (arrowhead indicates a junctional complex). (D) Channels are separated by loose connective tissue composed mostly of collagen (arrows). (E) Foci of mononuclear inflammatory cells are present (arrows). (F) Hemosiderin-laden macrophages (arrow) are among the inflammatory cells observed. Images are representative of 5 lesions from 3 Pdcd10 mice. Scale bars: $4 \mu \mathrm{m}$.
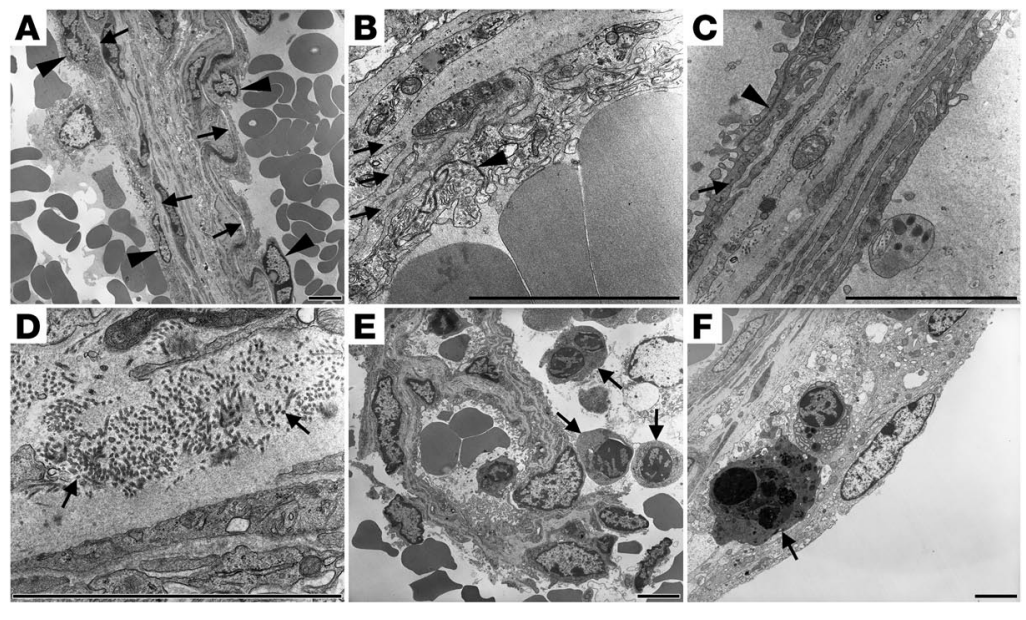

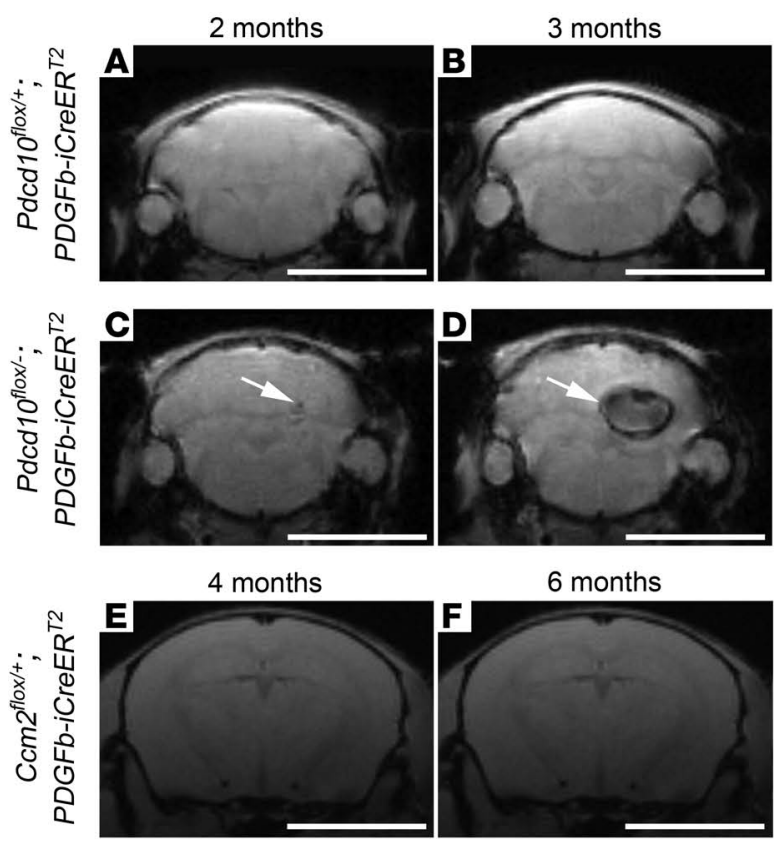

6 months
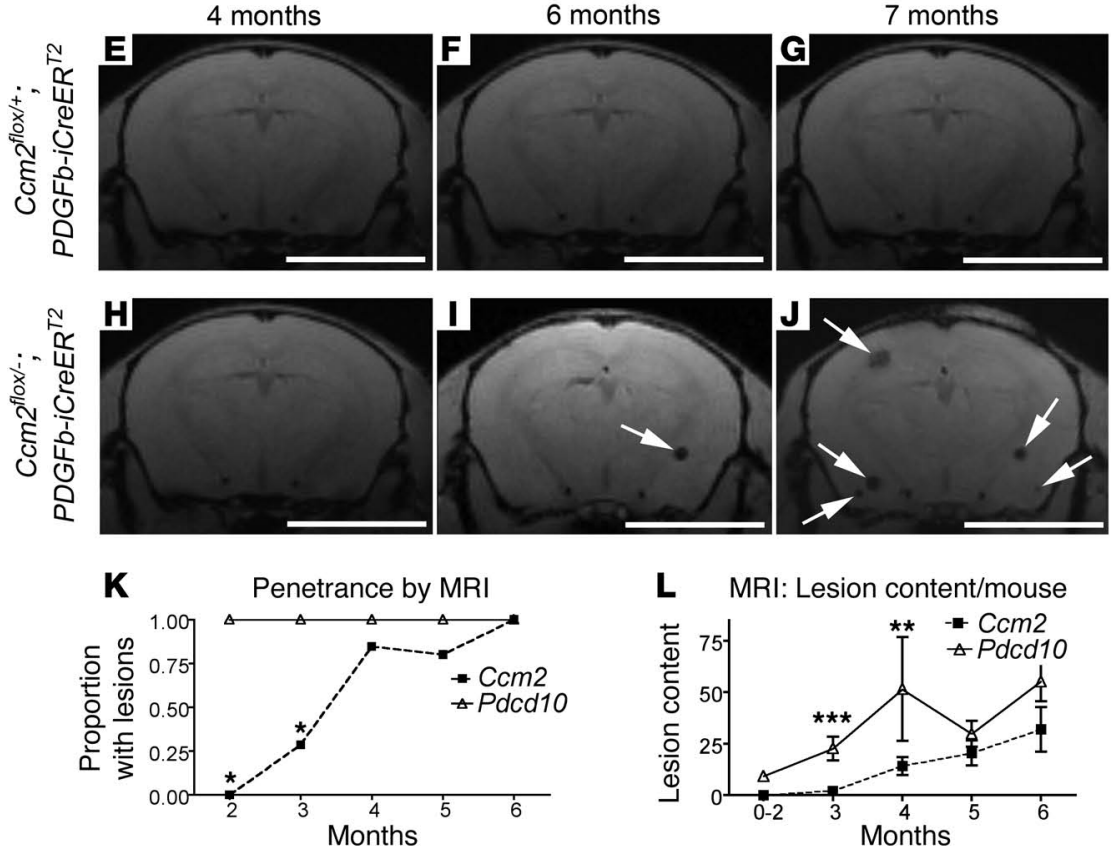

M MRI: Complex lesions/mouse
L MRI: Lesion content/mouse

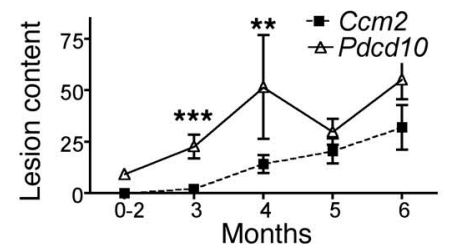

N CCM mice survival

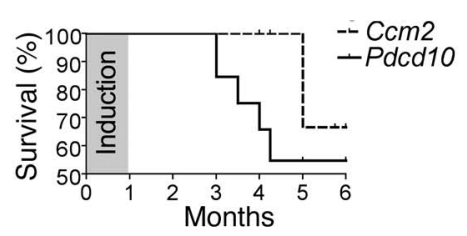

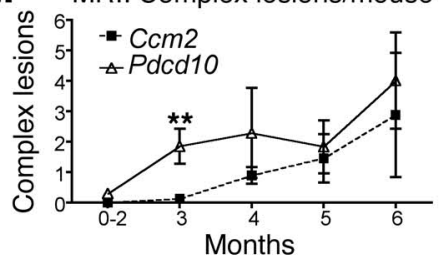

\section{Figure 7}

Natural history of murine CCM by MRI $-P d c d 10$ onsets earlier and is more severe than Ccm2. (A-D) Live MRI scans of the same Pdcd10 flox/+; $P D G F b$-iCreERT2 mouse at 2 months and 3 months (A and $\mathbf{B})$ and its $P d c d 10^{f l o x /-; P D G F b-}$ iCreER $R^{T 2}$ littermate (C and $\left.\mathbf{D}\right)$. Both mice were given tamoxifen at birth. (E-J) Live MRI scans of the same $\mathrm{Ccm} 2^{\text {flox/++}} ; P D G F b-i C r e E R^{T 2}$ mouse (E-G) and its $\mathrm{Ccm}^{\text {flox/-; }}$ PDGFb-iCreER ${ }^{\text {T2 }}$ littermate $(\mathbf{H}-\mathbf{J})$ at 4,6 , and 7 months. Both mice were given tamoxifen at birth. Arrows indicate CCM lesions. (K) Disease penetrance (proportion affected) by age in Ccm2 and Pdcd10 induced knockout mice as assessed by live MRI. (L) Lesion burden assessed as total number of lesions observed on each tomographic view (slice) of the MRI per mouse. (M) Number of complex lesions (lesions with bright cores) per mouse. (N) Kaplan-Meier survival curve of $\mathrm{Ccm} 2$ and $P d c d 10$ induced knockout mice. For $\mathbf{K}-\mathbf{N}$, $n=11 \mathrm{Ccm} 2, n=13 \mathrm{Pdcd} 10$. Data in $\mathbf{L}$ and $\mathbf{M}$ represent mean \pm SEM. ${ }^{*} P<0.01$; ${ }^{* *} P<0.05$; ${ }^{\star \star \star} P<0.001$. Scale bars: $1 \mathrm{~mm}$.

5'-TGAAAGAGGACATACTGGAGAGGC-3', primer Z: 5'-AAGGACAAGAAAGCACTGTTGACC-3'. Tamoxifen (Sigma-Aldrich) was resuspended in corn oil (Sigma-Aldrich), and $40 \mathrm{ng}$ was given as an i.p. injection to mouse pups on perinatal day 1 .

MRI of mouse brains. All MRI experiments were conducted on a 7T Bruker Biospec 70/30 USR scanner (Bruker Biospin MRI Inc.) equipped with a BGA12S gradients set. A combination of volume-transmitonly radio frequency coil (internal diameter: $72 \mathrm{~mm}$ ) and a quad-surface-receive-only coil (internal diameter: $1.5 \mathrm{~cm}$ ) were used. For live scans, mice were anesthetized in $2.5 \%$ isoflurane and then placed into the scanner on top of circulating heated water. During the scans, mice were monitored for temperature and respiration, with isoflurane concentration and water bath temperature adjusted to maintain a body temperature between $35.8^{\circ} \mathrm{C}$ and $37.6^{\circ} \mathrm{C}$ and respiration between 75 and 100 breaths $/ \mathrm{min}$. A gradient recalled echo sequence was used to acquire coronal slices spanning the whole brain. Sequence parameters were as follows: repetition time, $328 \mathrm{~ms}$; echo time, $5.4 \mathrm{~ms}$; flip-angle, $40^{\circ}$; 12 averages, in-plane-

mice, and PDGFb-iCreER ${ }^{T 2}$ mice were generously provided by Phil Soriano (Fred Hutchinson Cancer Research Center, Seattle, Washington, USA, and Mount Sinai School of Medicine, New York, New York, USA), Masashi Yanagisawa (University of Texas Southwestern Medical Center at Dallas, Dallas, Texas, USA), and Marcus Fruttiger (University College London Institute of Ophthalmology, London, United Kingdom) via Holger Gerhardt (London Research Institute - Cancer Research UK, London, United Kingdom), respectively. HPRT-Cre, Nestin-Cre, and Tomato-EGFP reporter mice were obtained from The Jackson Laboratory. Genotypes were determined by PCR analysis of genomic DNA isolated from either ear biopsies or yolk sac tissues using primers outlined in Supplemental Figure 1. Primer sequences were as follows: primer W: 5'-GCAATCCATCTTGTTCAATGGC-3', primer X: 5'-CGTAGGTCAGGGTGGTCACG-3', primer Y: resolution, $125 \mu \mathrm{m} \times 125 \mu \mathrm{m}$; and slice thickness, $0.5 \mathrm{~mm}$.

For postmortem specimens, mouse skulls were fixed in $4 \%$ formaldehyde for at least 3 days before the brain was imaged with the skull intact. Postmortem MRI scanning allows a high-resolution coverage of the brain at the expense of longer scan time. High-resolution 3D gradient echo was acquired using the same scanner configuration described above, with isotropic voxel size of $78 \mu \mathrm{m} \times 78 \mu \mathrm{m} \times 78 \mu \mathrm{m}$ over 9 hours. Other sequence parameters were as follows: repetition time, $250 \mathrm{~ms}$; echo time, $7.5 \mathrm{~ms}$; flip angle, $30^{\circ}$; and 2 averages.

Histology. Embryo and mouse CCM lesions were studied with antibodies to PECAM (1:250 dilution, clone MEC13.3; BD Biosciences) and PDCD10 (1:50 dilution, Prestige Antibody; Sigma-Aldrich). Improved visualization on paraffin sections was obtained using a biotinylated tyramide signal 
A

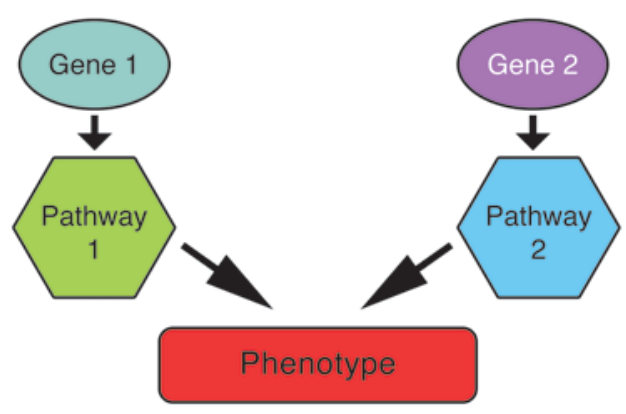

B

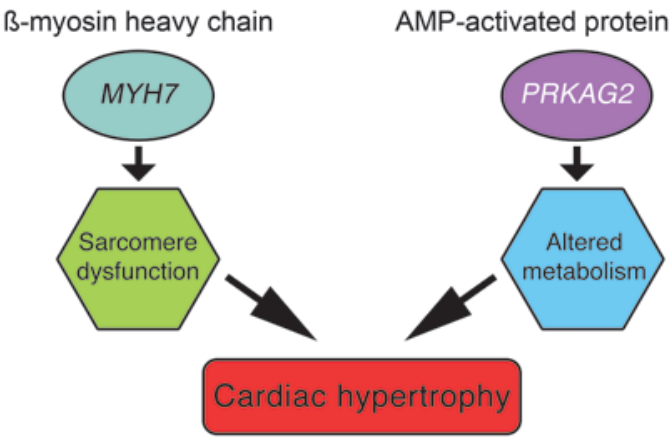

C

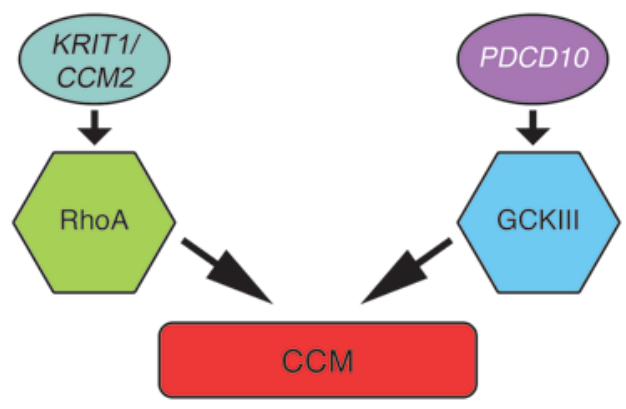

amplification (TSA) kit (PerkinElmer) according to the manufacturer's instructions. To demonstrate tissue specificity of PDFGb-iCreER ${ }^{T 2}$, brains from mice carrying a $L a c Z$ reporter allele were fixed and then stained with X-gal as previously described (48). Brains from mice carrying the Tomato-EGFP reporter allele were fixed and then embedded into OCT (Fisher Scientific) for fluorescent frozen sections. H\&E staining of embryos was performed using standard procedures.

For histology of adult mouse brains, mouse skulls were fixed in $4 \%$ formaldehyde for at least 3 days or zinc-buffered formalin overnight before the brain was removed and sliced into 4 coronal sections. These sections were embedded into paraffin by standard techniques. Prussian blue staining for iron and Masson's trichrome staining of mouse brain sections were performed using the Artisan system (DAKO) according to manufacturer's instructions. Staining for elastin, laminin, and collagen IV was performed by the Immunohistochemistry Research and Development Lab at ARUP Laboratories. Human CCM samples were stained in the same way as adult mouse brains except for CD34 staining, which was performed by the Central Labs at Intermountain Medical Center (Murray, Utah, USA).

Fluorescent staining of mouse retinas. Mice were anesthetized using Avertin (2,2,2-tribromoethanol, $0.4 \mathrm{mg} / \mathrm{g}$; Acros Organics) and perfused with fluorescently conjugated tomato lectin (Vector Labs) as previously described (49) at a dose of $5 \mu \mathrm{g} / \mathrm{g}$ body weight. After 5 minutes, mice were perfused with saline and then with $4 \%$ formaldehyde. Retinas were then extracted, stained, and mounted as previously described (50).

\section{Figure 8}

Convergence of different mechanistic pathways in common pathology. (A) Proposed schema for different genes acting on separate mechanistic pathways, yet ultimately resulting in a common expression of disease. (B) Genetic studies of hypertrophic cardiomyopathy highlight genes that can be grouped broadly into 2 separate mechanistic pathways: sarcomeric proteins such as $\beta$-myosin heavy chain ( $\mathrm{MYH} 7$ ), and metabolic genes including adenosine monophosphate-activated protein kinase (PRKAG2). (C) Studies of mouse development, cell biology, and signaling suggest that KRIT1 and CCM2 signal through RhoA GTPase, while PDCD10 signals through GCKIII kinases to lead to cavernous malformations.

Analysis of mouse embryos. Confocal immunofluorescence of embryos and fetal ultrasound were performed as previously described (9).

Transmission electron microscopy. Samples were fixed in $1 \%$ formaldehyde, $2.5 \%$ glutaraldehyde in $0.1 \mathrm{M}$ sodium cacodylate buffer with $8 \mathrm{mM} \mathrm{CaCl}_{2}$ and $2.4 \%$ sucrose, $\mathrm{pH} 7.4$, for at least 3 days. Samples were then rinsed in $0.1 \mathrm{M}$ cacodylate buffer, postfixed in $2 \%$ osmium tetroxide in $0.1 \mathrm{M}$ cacodylate buffer, rinsed in type I water, and then enbloc stained with saturated aqueous uranyl acetate. Samples were dehydrated through a graded series of ethanols, followed with final dehydration in acetone, and then infiltrated and embedded in an epoxy resin.

Thick sections and thin sections were cut on a Leica EM UC6 ultramicrotome (Leica Microsystems). The thick sections were cut with glass and the thin sections were cut with a diatome diamond knife. Thick sections were dried on glass slides and were stained with $1 \%$ toluidine blue $\mathrm{O}$ in $1 \%$ aqueous sodium borate. Thin sections were placed on 135 mesh copper hex grids and stained sequentially with saturated aqueous uranyl acetate and Reynolds lead citrate.

Sections were examined on an FEI Tecnai T-12 transmission electron microscope (FEI) at $80 \mathrm{KV}$. Images were taken on Kodak 4489 electron microscope film, developed with Kodak D-19 developer, fixed, washed, and dried. The negatives were scanned on an Epson Perfection Photo 4990 flatbed scanner.

Cell culture. HUVEC and HMVEC were obtained from Lonza and grown according to the manufacturer's instructions in EGM-2 media (HUVEC) or EGM-2MV media (HMVEC). Human embryonic kidney (HEK 293T) cells (ATCC) were grown in DMEM (Gibco; Invitrogen) with $10 \%$ fetal bovine serum (Bio-West) supplemented with antibiotics. EAHY cells were generously provided by Andrew Weyrich (University of Utah) and were grown in DMEM with $10 \%$ fetal bovine serum and HAT supplement (Sigma-Aldrich).

Transfection of ECs with siRNAs. Human CCM2 and PDCD10 siRNAs were obtained from Dharmacon. Luciferase GL2 duplex or nontargeting siRNA (Dharmacon) were used as controls. EC transfection with siRNAs was carried out in growth media with $1 \%$ serum. Details of the siRNA transfection protocol have been described previously (51).

$R T-P C R$. Total RNA was extracted from EC vasculogenesis assay at indicated time points or from siRNA-treated ECs using the ToTALLY RNA Isolation Kit (Ambion) according to the manufacturer's instructions. RNA $(1 \mu \mathrm{g})$ was reverse transcribed using AccuScript High Fidelity 1st strand cDNA synthesis kit (Stratagene; Agilent). RT-PCR amplification used the following primers: forward: 5'-AGACTTCACGGAGTCCCTTC-3', reverse: 5'-AGAAGGTCTGAAGTATTAAGTTGG-3'. For quantitative real-time PCR, total RNA was extracted from cultured endothelial cells or from embryos using the NucleoSpin RNA II Kit (Clontech) according to the manufacturer's instructions. Reverse transcription was performed with random primers using the RETROScript Kit (Ambion). Quantitative PCR was performed with TaqMan assays (Applied Biosystems) for human CCM2, PDCD10, GAPDH, STK24, STK25, and MST4 or mouse Pdcd10 and Gapdh. Quantification was performed by standard curve method, and 
CCM2 and PDCD10 transcripts were normalized to GAPDH for comparisons. RT-PCR primers for embryonic $P d c d 10$ transcripts were as follows: primer 4F: 5'-TTCACCGAGTCCCTCCTTCG-3'; primer 7/8R: 5'-GAACACATTTATTGCCTTGCCATC-3'; primer 1F: 5'-AAGTCCGTGCCTCAGTTGCC-3'; and primer EGFP-R: 5'-TCCTCGCCCTTGCTCACC-3'.

Immunofluorescent cell staining. Glass chamber slides (Nalge Nunc; Thermo Fisher) were coated with human fibronectin (Biomedical Technologies Inc.), and transfected cells were seeded at 50,000 cells per well. Cells were fixed in $4 \%$ formaldehyde and actin cytoskeleton was visualized using fluorescently conjugated phalloidin (Molecular Probes; Invitrogen). Images were obtained with an Olympus FV300 confocal microscope.

Endothelial cell vasculogenesis in $3 D$ collagen matrices. This assay was performed as previously described $(9,38)$.

Immunoprecipitation and Western blotting. An expressed sequence tag (EST) for human PDCD10 (IMAGE: 3050540) was obtained from ATCC and cloned into a pcDNA3.1 $\mathrm{Hygro}^{+}$plasmid (Invitrogen) modified to encode an N-terminal FLAG tag. ESTs for Drosophila Pdcd10 and GCKIII were obtained from the Drosophila Genomics Resource Center and cloned into a pcDNA3.1 Hygro $^{+}$plasmid modified to encode an N-terminal FLAG tag (Pdcd10) or HA tag (GCKIII). PDCD $10 \Delta 18$ constructs were made using nested PCR with primers designed to overlap and omit the 54 nucleotides encoding those 18 amino acids. Plasmids were transfected into EAHY or HEK 293T cells using Lipofectamine 2000 (Invitrogen) according to the manufacturer's instructions, with a reduced dosage of DNA and lipofectamine for EAHY cells. Two days after transfection, cells were scraped into lysis buffer $(50 \mathrm{mM} \mathrm{Tris-} \mathrm{HCl}$ at $\mathrm{pH} 7.5,100 \mathrm{mM} \mathrm{NaCl}, 0.5 \%$ Triton X-100) supplemented with protease and phosphatase inhibitors and $1 \mathrm{mM}$ DTT. A portion of cell lysate was retained as whole cell lysate and the rest precleared with normal mouse IgG conjugated to agarose beads (Santa Cruz Biotechnology Inc.) at $4^{\circ} \mathrm{C}$ for 1 hour, then incubated with anti-FLAG resin at $4^{\circ} \mathrm{C}$ for 2 hours. The beads were washed 3 times with lysis buffer, and bound proteins were eluted using Laemmli sample buffer. Presence of FLAG-tagged PDCD10 proteins was detected using the anti-FLAG M2 antibody (Sigma-Aldrich). Presence of HA-tagged GCKIII was detected using an anti-HA antibody (Santa Cruz Biotechnology Inc.). Nearinfrared secondary antibodies (LI-COR Biosciences) were used to probe the blots, which were visualized using the Odyssey system (LI-COR Biosciences).

Rabbit polyclonal antibody against PDCD10 was from Proteintech Group. Rabbit antibodies against phospho-myosin light chain (Thr18/Ser19), phospho-VEGFR2 (Tyr1175), total VEGFR2, phospho-PLC $\gamma$ (Tyr783), total PLC $\gamma$, phospho-ERK1/2 (Thr202/Tyr204), and total ERK1/2 were from Cell Signaling Technology. $\alpha$-Actinin antibody (clone H-2) was from Santa Cruz Biotechnology Inc. HRP-conjugated secondary antibodies were from Jackson ImmunoResearch. Western blots were developed using the ECL Plus Western blotting reagent (GE Healthcare) and Kodak Biomax MR film.

Drosophila strains. The GAL4/UAS system was used for tissue-specific RNAi knockdown (43). The driver used was btl-GAL4 (42), and RNAi targeting constructs v109453 (against $P d c d 10$ ) (41), v106841 (against $P d c d 10$ ) (41), and v49559 (against GCKIII) (44) were obtained from the Vienna Drosophila RNAi Center. For rescue experiments, full-length cDNAs for Drosophila Pdcd10 were cloned into a pUAST transformation vector containing an attb site for site-specific transformation (52) provided by Carl Thummel (University of Utah). Transgenes were injected into docking sites VK00027 (53) and attp0016 (52) by Genetic Services Inc., and homozygous lines were established by standard methods.

For cDNA rescue experiments, flies homozygous for the cDNA rescuing construct on the third chromosome were crossed to a homozygous btl-GAL4, UAS-GFP driver line on the second chromosome to generate transheterozygote flies (btl-GAL4,UAS-GFP/+; UAS-Rescue/+). Males of this genotype were then crossed to virgin females homozygous for the RNAi targeting construct located on the second chromosome. GFP expression indicated the presence of the btl-GAL4, UAS-GFP transgenes. All of these larvae contain 1 copy of the RNAi targeting construct, and half also have the cDNA rescue transgene. Results shown used the v109453 line, which targets the 3' UTR of Pdcd10. The v106841 line showed similar results.

Analysis of tracheal tube formation in Drosophila larvae. Animals were scored at the prewandering third instar larval stage for tracheal defects. Animals were graded by scoring air filling in primary sprouts emanating from the dorsal trunk. All primary sprouts were scored bilaterally (at least 12 per larva). The animals were categorized as having severe, moderate, or mild defects or wild-type if they had 3 or more of the following defects: (a) severe defects were truncations before the first bifurcation of the primary side branches or immediately after branching; (b) moderate defects represented gaps in air filling with filled tubes on both sides; (c) mild defects were loss of air filling only in the fine terminal tubes. In the event that more than 1 criterion was met, the more severe category was selected. If animals had fewer than 3 branches with a given defect, the less severe category was selected. Only animals with no defects were categorized as wild type.

Mouse and human experiments. All mouse experiments were approved by the University of Utah Institutional Animal Care and Use Committee. Human tissue samples were obtained with written informed consent and provided by Connie Lee and Amy Akers (Angioma Alliance, Norfolk, Virginia, USA) and Randy Jensen (University of Utah). Human experiments were approved by the Institutional Review Board of the University of Utah.

Statistics. For actin stress fiber content, and for in vitro lumen formation with GCKIII kinases, we performed ANOVA with Tukey's post hoc analysis with an $\alpha$ value of 0.05 . For in vitro lumen formation time course for PDCD10, we performed statistical comparisons between treatment groups with a 2-tailed paired-sample $t$ test with an $\alpha$ value of 0.05 . For the tracheal tube formation rescue experiment, we performed a $\chi^{2}$ test for independence with an $\alpha$ value of 0.05 (degrees of freedom $=9$ ). For CCM penetrance and lesion content, we performed a 2-tailed $t$ test with an $\alpha$ value of 0.05 .

\section{Acknowledgments}

We thank N. London, S. Navankasattusas, L. Shi, Y. Xiong, C. Jensen, J. Zhu, D. Zurcher, A. Fang, T. Mleynek, and D. Lim for technical assistance; O. Abdullah and E. Hsu and the University of Utah Small Animal Imaging Facility; C. Rodesch and the University of Utah Cell Imaging/Fluorescence Facility; N. Chandler and the University of Utah Electron Microscopy Facility; S. Tripp and the Immunohistochemistry Research and Development Lab at ARUP Laboratories; J. Hansen at Central Labs at Intermountain Medical Center; K. Thomas and S. Odelberg for critical comments and helpful scientific discussions; and S. Chin for helpful scientific discussions. This work was funded by the US NIH (to G.E. Davis, M.M. Metzstein, K.J. Whitehead, and D.Y. Li), including training grant T32-GM007464 (to A.C. Chan and O.E. Ruiz), the Hellenic Cardiological Society (to N.A. Diakos), the American Heart Association (to K.J. Whitehead and D.Y. Li), the H.A. and Edna Benning Foundation, the Juvenile Diabetes Research Foundation, and the Burroughs Wellcome Fund (to D.Y. Li).

Received for publication July 16, 2010, and accepted in revised form March 2, 2011.

Address correspondence to: Kevin J. Whitehead, Room 4A100, $30 \mathrm{~N}$ 1900 East, Salt Lake City, Utah 84132, USA. Phone: 801.581.7715; Fax: 801.581.7735; E-mail: kevin.whitehead@u2m2.utah.edu. Or to: Dean Y. Li, Building 533 Room 4220, 15 N 2030 East, Salt Lake City, Utah 84112, USA. Phone: 801.585.5505; Fax: 801.585.0701; E-mail: dean.li@u2m2.utah.edu. 
1. Otten P, Pizzolato GP, Rilliet B, Berney J. A propos de 131 cas d'angiomes caverneux (cavernomes) du S.N.C. repérés par l'analyse rétrospective de 24535 autopsies. Neurochirurgie. 1989;35(2):82-83.

2. Vernooij $\mathrm{MW}$, et al. Incidental findings on brain MRI in the general population. $N$ Engl J Med. 2007;357(18):1821-1828.

3. Hasegawa T, McInerney J, Kondziolka D, Lee JY, Flickinger JC, Lunsford LD. Long-term results after stereotactic radiosurgery for patients with cavernous malformations. Neurosurgery. 2002;50(6):1190-1197.

4. Chappell PM, Steinberg GK, Marks MP. Clinically documented hemorrhage in cerebral arteriovenous malformations: MR characteristics. Radiology. 1992;183(3):719-724.

5. Burger PC, Scheithauer BW. Tumors of the Central Nervous System. Washington, DC, USA: American Registry of Pathology; 2007.

6. Wang H, Gujrati M. Pathology of cerebral cavernous malformations. In: Lanzino G, Spetzler RF, eds. Cavernous Malformations of the Brain and Spinal Cord. New York, New York, USA: Thieme Medical Publishers, Inc; 2008:22-25.

7. Faurobert E, Albiges-Rizo C. Recent insights into cerebral cavernous malformations: a complex jigsaw puzzle under construction. FEBS J. 2010;277(5):1084-1096.

8. Hilder TL, et al. Proteomic identification of the cerebral cavernous malformation signaling complex. J Proteome Res. 2007;6(11):4343-4355.

9. Whitehead KJ, et al. The cerebral cavernous malformation signaling pathway promotes vascular integrity via Rho GTPases. Nat Med. 2009;15(2):177-184.

10. Whitehead KJ, Plummer NW, Adams JA, Marchuk DA, Li DY. Ccm 1 is required for arterial morphogenesis: implications for the etiology of human cavernous malformations. Development. 2004; 131(6):1437-1448

11. Boulday $G$, et al. Tissue-specific conditional CCM2 knockout mice establish the essential role of endothelial CCM2 in angiogenesis: implications for human cerebral cavernous malformations. Dis Model Mech. 2009;2(3-4):168-177.

12. Stockton RA, Shenkar R, Awad IA, Ginsberg MH. Cerebral cavernous malformations proteins inhibit Rho kinase to stabilize vascular integrity. J Exp Med. 2010;207(4):881-896

13. Glading A, Han J, Stockton RA, Ginsberg MH. KRIT-1/CCM1 is a Rap1 effector that regulates endothelial cell cell junctions. J Cell Biol. 2007; 179(2):247-254.

14. Borikova AL, et al. Rho kinase inhibition rescues the endothelial cell cerebral cavernous malformation phenotype. J Biol Chem. 2010;285(16):11760-11764.

15. Zawistowski JS, et al. CCM1 and CCM2 protein interactions in cell signaling: implications for cerebral cavernous malformations pathogenesis. Hum Mol Genet. 2005;14(17):2521-2531.

16. Fidalgo M, Fraile M, Pires A, Force T, Pombo C, Zalvide J. CCM3/PDCD10 stabilizes GCKIII proteins to promote Golgi assembly and cell orientation. J Cell Sci. 2010;123(pt 8):1274-1284.

17. Zhang J, Clatterbuck RE, Rigamonti D, Chang DD, Dietz HC. Interaction between krit1 and icap1alpha infers perturbation of integrin beta1mediated angiogenesis in the pathogenesis of cerebral cavernous malformation. Hum Mol Genet. 2001;10(25):2953-2960.

18. Zawistowski JS, Serebriiskii IG, Lee MF, Golemis EA, Marchuk DA. KRIT1 association with the integrin-binding protein ICAP-1: a new direction in the elucidation of cerebral cavernous malformations (CCM1) pathogenesis. Hum Mol Genet. 2002;11(4):389-396.

19. Uhlik MT, et al. Rac-MEKK3-MKK3 scaffolding for p38 MAPK activation during hyperosmotic shock. Nat Cell Biol. 2003;5(12):1104-1110.

20. Ma X, et al. PDCD10 interacts with Ste20-related kinase MST4 to promote cell growth and transformation via modulation of the ERK pathway. Mol Biol Cell. 2007;18(6):1965-1978.

21 . Voss K, et al. Functional analyses of human and zebrafish 18-amino acid in-frame deletion pave the way for domain mapping of the cerebral cavernous malformation 3 protein. Hum Mutat. 2009;30(6):1003-1011.

22. Goudreault M, et al. A PP2A phosphatase high-density interaction network identifies a novel striatininteracting phosphatase and kinase complex linked to the cerebral cavernous malformation 3 (CCM3) protein. Mol Cell Proteomics. 2008;8(1):157-171.

23. Zheng $X$, et al. CCM3 signaling through sterile 20like kinases plays an essential role during zebrafish cardiovascular development and cerebral cavernous malformations. J Clin Invest. 2010;120(8):2795-2804.

24. Denier C, et al. Genotype-phenotype correlations in cerebral cavernous malformations patients. Ann Neurol. 2006;60(5):550-556.

25. Sirvente J, Enjolras O, Wassef M, Tournier-Lasserve E, Labauge P. Frequency and phenotypes of cutaneous vascular malformations in a consecutive series of 417 patients with familial cerebral cavernous malformations. J Eur Acad Dermatol Venereol. 2009;23(9):1066-1072.

26. Labauge $P$, et al. Multiple dural lesions mimicking meningiomas in patients with CCM3/PDCD10 mutations. Neurology. 2009;72(23):2044-2046.

27. Akers AL, Johnson E, Steinberg GK, Zabramski JM, Marchuk DA. Biallelic somatic and germline mutations in cerebral cavernous malformations (CCMs): evidence for a two-hit mechanism of CCM pathogenesis. Hum Mol Genet. 2009;18(5):919-930.

28. Gault J, et al. Cerebral cavernous malformations: somatic mutations in vascular endothelial cells. Neurosurgery. 2009;65(1):138-144

29. Pagenstecher A, Stahl S, Sure U, Felbor U. A twohit mechanism causes cerebral cavernous malformations: complete inactivation of CCM1, CCM 2 or CCM3 in affected endothelial cells. Hum Mol Genet. 2009;18(5):911-918.

30. Rigamonti D, et al. Cerebral cavernous malformations. Incidence and familial occurrence. $N$ Engl J Med. 1988;319(6):343-347.

31. Labauge P, Brunereau L, Levy C, Laberge S, Houtteville JP. The natural history of familial cerebral cavernomas: a retrospective MRI study of 40 patients. Neuroradiology. 2000;42(5):327-332.

32. Del Curling O Jr, Kelly DL Jr, Elster AD, Craven TE. An analysis of the natural history of cavernous angiomas. J Neurosurg. 1991;75(5):702-708.

33. Knudson AG Jr. Mutation and cancer: statistical study of retinoblastoma. Proc Natl Acad Sci U S A. 1971;68(4):820-823.

34. Gault J, Shenkar R, Recksiek P, Awad IA. Biallelic somatic and germ line CCM1 truncating mutations in a cerebral cavernous malformation lesion. Stroke. 2005;36(4):872-874.

35. Shenkar R, et al. Advanced magnetic resonance imaging of cerebral cavernous malformations: part II. Imaging of lesions in murine models. Neurosurgery. 2008;63(4):790-797.

36. McDonald DA, et al. A novel mouse model of cere- bral cavernous malformations based on the two-hit mutation hypothesis recapitulates the human disease. Hum Mol Genet. 2011;20(2):211-222.

37. Claxton S, Kostourou V, Jadeja S, Chambon P, Hodivala-Dilke K, Fruttiger M. Efficient, inducible Cre-recombinase activation in vascular endothelium. Genesis. 2008;46(2):74-80.

38. Davis GE, Camarillo CW. An alpha 2 beta 1 integrin-dependent pinocytic mechanism involving intracellular vacuole formation and coalescence regulates capillary lumen and tube formation in three-dimensional collagen matrix. Exp Cell Res. 1996;224(1):39-51.

39. Ghabrial A, Luschnig S, Metzstein MM, Krasnow MA. Branching morphogenesis of the Drosophila tracheal system. Annu Rev Cell Dev Biol. 2003;19:623-647.

40. Manning G, Krasnow MA. Development of the Drosophila tracheal system. In The Development of Drosophila melanogaster. Bate M, Martinez Arias A, eds. Plainview, New York, USA: Cold Spring Harbor Laboratory Press; 1993:609-685.

41. Mummery-Widmer JL, et al. Genome-wide analysis of Notch signalling in Drosophila by transgenic RNAi. Nature. 2009;458(7241):987-992.

42. Shiga Y, Tanaka-Matakatsu M, Hayashi S. A nuclear GFP/beta-galactosidase fusion protein as a marker for morphogenesis in living Drosophila. Dev Growth Differ. 1996;38(1):99-106

43. Brand AH, Perrimon N. Targeted gene expression as a means of altering cell fates and generating dominant phenotypes. Development. 1993;118(2):401-415.

44. Dietzl G, et al. A genome-wide transgenic RNAi library for conditional gene inactivation in Drosophila. Nature. 2007;448(7150):151-156.

45. Clatterbuck RE, Eberhart CG, Crain BJ, Rigamonti D. Ultrastructural and immunocytochemical evidence that an incompetent blood-brain barrier is related to the pathophysiology of cavernous malformations. J Neurol Neurosurg Psychiatry. 2001;71(2):188-192.

46. Wang L, Seidman JG, Seidman CE. Narrative review: harnessing molecular genetics for the diagnosis and management of hypertrophic cardiomyopathy. Ann Intern Med. 2010;152(8):513-520.

47. He Y, et al. Stabilization of VEGFR2 signaling by cerebral cavernous malformation 3 is critical for vascular development. Sci Signal. 2010;3(116):ra26.

48. Navankasattusas $S$, et al. The netrin receptor UNC5B promotes angiogenesis in specific vascular beds. Development. 2008;135(4):659-667.

49. di Tomaso E, et al. PDGF-C induces maturation of blood vessels in a model of glioblastoma and attenuates the response to anti-VEGF treatment. PLOS ONE. 2009;4(4):e5123.

50. Jones CA, et al. Robo4 stabilizes the vascular network by inhibiting pathologic angiogenesis and endothelial hyperpermeability. Nat Med. 2008;14(4):448-453.

51. Saunders WB, Bayless KJ, Davis GE. MMP-1 activation by serine proteases and MMP-10 induces human capillary tubular network collapse and regression in $3 \mathrm{D}$ collagen matrices. J Cell Sci. 2005;118(pt 10):2325-2340.

52. Groth AC, Fish M, Nusse R, Calos MP. Construction of transgenic Drosophila by using the sitespecific integrase from phage phiC31. Genetics. 2004;166(4):1775-1782.

53. Venken KJ, He Y, Hoskins RA, Bellen HJ. P[acman]: a BAC transgenic platform for targeted insertion of large DNA fragments in D. melanogaster. Science. 2006;314(5806):1747-1751 